\title{
Arqueologías globales en la literatura cubana: de las ruinas al chicle
}

Global Archaeologies in Cuban Literature: From Ruins to Bubble Gum Arqueologias globais na literatura cubana: das ruínas ao chiclete

\section{Catalina Quesada Gómez}

UNIVERSITY OF MIAMI, ESTADOS UNIDOS

Profesora de Literatura Latinoamericana en University of Miami,

Estados Unidos. Doctora en Letras, Universidad de Sevilla, España.

Autora de La metanovela hispanoamericana en el último tercio

del siglo XX. Las prácticas metanovelescas de Salvador Elizondo,

Severo Sarduy, Fosé Donoso y Ricardo Piglia (Arco/Libros, 2009)

y Literatura y globalización: la narrativa hispanoamericana

en el siglo XXI (espacio, tiempo, géneros) (Universidad de

Antioquia, 2014). Editora del dossier Cultura y globalización en

Hispanoamérica en la revista Pasavento: Revista de Estudios

Hispánicos (2014). Correo electrónico: cquesadag@miami.edu

Documento accesible en línea desde la siguiente dirección: http://revistas.javeriana.edu.co 


\section{Resumen}

Este artículo estudia el papel de ciertos objetos o materiales en la obra de los autores cubanos Jorge Enrique Lage y Legna Rodríguez Iglesias, para dilucidar, primero, cómo acontece el distanciamiento respecto a la generación anterior a partir del uso de dichos materiales, que les sirven para abandonar los lugares comunes de la carencia, la decadencia o las ruinas, y manifestar así su desapego en relación con una literatura preocupada en exceso por la temática nacional, y, segundo, cómo el interés central por lo material en estos escritores no desemboca obligatoriamente en un nuevo realismo.

Palabras clave: literatura cubana; cultura material; literatura y globalización; Jorge Enrique Lage; Legna Rodríguez Iglesias

\section{Abstract}

This article examines the role of certain objects or materials in the works of Cuban writers Jorge Enrique Lage and Legna Rodríguez Iglesias. It aims at clarifying, first, the way they distance themselves from the previous generation by using those materials. Lage and Rodríguez Iglesias abandon the clichés of scarcity, decay or ruins, and thus express their detachment from a literature obsessed with national topics. Furthermore, the essay analyzes how the focus on the material does not lead necessarily to a new realism in these writers.

Keywords: Cuban literatura; material culture; literature and globalization; Jorge Enrique Lage; Legna Rodríguez Iglesias

\section{Resumo}

Este artigo estuda o papel de certos objetos ou materiais na obra dos autores cubanos Jorge Enrique Lage e Legna Rodríguez Iglesias, para esclarecer, primeiro, como acontece o distanciamento no que diz respeito da geração anterior a partir do uso de tais materiais, que lhes servem para abandonar os lugares comuns da carência, a decadência ou as ruinas, e manifestar assim seu desapego no que diz respeito de uma literatura preocupada demais pela temática nacional, e, segundo, como o interesse central pelo material nesses escritores não desemboca obrigatoriamente em um novo realismo.

Palavras-chave: literatura cubana; cultura material; literatura e globalização; Jorge Enrique Lage; Legna Rodríguez Iglesias

RECIBIDO: 10 DE OCTUBRE DE 2015. ACEPTADO: 11 DE NOVIEMBRE DE 2015. DISPONIBLE EN LÍNEA: 1 DE JULIO DE 2016

\section{Cómo citar este artículo:}

Quesada Gómez, Catalina. "Arqueologías globales en la literatura cubana: de las ruinas al chicle". Cuadernos de Literatura 20.40 (2016): 313-

324. http://dx.doi.org/10.11144/Javeriana.cl20-40.aglc 
Si HAY UN espacio en América Latina en el que los estudios sobre cultura material ofrecen inmensas y renovadas posibilidades es, sin lugar a dudas, la Cuba contemporánea. Desde la particular obsesión cubana por los alimentos a los más recientes estudios alrededor de lo postsoviético a partir de determinados objetos (y la consiguiente búsqueda de raigambre arqueológica de "lo soviético" en los años precedentes), por citar tan solo dos ejemplos en los que los estudios sobre cultura material pueden resultar especialmente productivos, vemos que la isla se presenta como una viva almáciga de cosas tangibles que han servido para trazar el imaginario de la cultura cubana (la cubanidad) y, en ocasiones, definir lo nacional (o lo posnacional).

En líneas generales, la literatura contemporánea escrita por cubanos ha girado obsesivamente en torno a Cuba, su insularidad o su carácter diaspórico y, más recientemente - en autores que comienzan a publicar en los años noventa-, en torno a la condición posnacional o postsoviética (Loss y Prieto) de dicha sociedad. Baste citar como ejemplos paradigmáticos de lo último las respectivas obras de José Manuel Prieto o Antonio José Ponte. Sin embargo, como apunta Rafael Rojas, bien entrado el siglo XXI surgen escritores que ya no están interesados en el cuestionamiento ideológico de lo nacional, sino que se adentran en una "ficción global" que "se produce desde nuevas comunidades conectadas e intercambiables, que ya no se piensan como aisladas o excepcionales" (Rojas) y que, abandonando o subvirtiendo los lugares comunes de la carencia, la decadencia o las ruinas (Birkenmaier y Whitfield), construyen obras cuyo centro, presente o ausente, no es necesariamente Cuba. Aquí analizo el papel de ciertos objetos o materiales en la obra de autores nacidos a finales de los años setenta $u$ ochenta, como Jorge Enrique Lage o Legna Rodríguez (aunque sería extensible a otros como Ahmel Echeverría u Osdany Morales), para dilucidar no solo cómo acontece ese distanciamiento respecto a la generación anterior, sino también cómo en estos escritores el interés central por lo material (o por la materialidad de la escritura) no desemboca obligatoriamente en un nuevo realismo.

Raúl Rubio, en "Cosas cubanas", ha examinado toda una serie de objetos, cosas tangibles o productos de mercadotecnia que tanto en Cuba como fuera de ella se han utilizado en el siglo XX como indicadores de cubanidad. En la segunda mitad del siglo, se habría recurrido, por un lado, al mito de la Revolución, como alegoría de la fundación nacional; por otro, al mito de la carencia y la nostalgia por la Cuba BC (before Castro), esto último especialmente llevado a cabo por los exiliados (en particular los denominados hyphenated, esto es, los Cuban-American) y su particular consumo de café, guayaberas y memorabilia de toda índole: 
I demonstrate here that these objects are vessels representative of the established national label "Cuban," what may be considered an imaginary myth, correlated to what Antoni Kapcia proposes in his book Cuba: Island of Dreams. Nevertheless, there are tangible themes within this label that can be summarized as a composite of ideas pertaining to national identities, social characteristics, history, and those significant of the ideology and politics pertinent to the Cuban nation. My proposal here departs from the idea that consumption on the island consists of limited government rationing, a weak Cuban currency that has been dependent on dollarization, and an underground black market of mercantile material needs (food, clothes) and materials wants (video movies, internet access, brand name clothes, toys, etc.). Meanwhile, a strong marketplace outside the island thrives on manufacturing Cuban-identified materials in a transnational context, for example, Cuban-style guayaberas, designed in Cuban Miami and manufactured in Taiwan. Many of these products are designed on established allegories about what is considered "Cuban." In turn, these products are commercialized through multiple transnational transpositions of globalization, sold in diverse locales, and dependent on a wide variety of consumer tastes which create a demand for them. (Rubio, "Materializing Havana" 163)

Desde la música hasta el ron, el daiquiri y los mojitos pasando, claro está, por el azúcar, el café y el tabaco, Rubio traza un amplio mapa de lo que denomina la cultura material cubana, así como del modo en que esta ha sido y es percibida (y, sobre todo, consumida) fuera de la isla y dentro de ella. Como sabemos, la literatura y el cine han recurrido con frecuencia a esos objetos para recrear el imaginario de la cultura cubana, moldear el concepto de nación y perfilar la cubanidad.

En relación con la literatura reciente (no la última, sino la penúltima), es necesario tener en cuenta lo que Odette Casamayor-Cisneros ha llamado ingravidez postsoviética - que ella analiza en la obra de Ena Lucía Portela, Pedro de Jesús, Gerardo Fernández Fe, Yohamna Depestre, Wendy Guerra y Orlando Pardo Lazo-y que se apoya, en lo esencial, en la sustitución del desencanto de la generación anterior por la incertidumbre: "La incertidumbre constituye pues el factor distintivo de estos creadores para quienes todo se tornaría incierto: no sólo su propia existencia sino la de la nación, la revolución, el socialismo" (35). Dicha ingravidez, en la que la desilusión con la realidad está tan ausente como la nostalgia regresiva precastrista, se acentúa en los autores que aquí comentamos, cuya obra late en mayor medida a la hora del mundo, dejando completamente al margen la sempiterna excepcionalidad cubana y la preocupación social o nacional. 
Del mismo modo, todos los rasgos que ella vincula al sujeto ingrávido - la suspensión ética, el carácter flotante de los espacios representados, el desinterés por definir la cubanidad - y que entroncan evidentemente tanto con las teorías de Jameson sobre la posmodernidad como con las de Bauman acerca de la modernidad líquida se intensifican notablemente y se radicalizan en los autores más jóvenes. Sus obras ostentan, además, una peculiaridad: el contraste entre dicha ingravidez y la recurrencia obsesiva a lo material, bajo la forma, por un lado, de materias primas o listas de objetos, fastuosamente erigidos en el centro del texto literario; por otro, por la insistencia en la materialidad de la escritura, una autorreferencialidad que ya había sido enarbolada por los autores cubanos en los años noventa (Casamayor-Cisneros 54), por no mencionar a escritores considerados canónicos, como Severo Sarduy.

Sin embargo, pese al desinterés por los problemas en la Cuba contemporánea que caracterizaría la obra de los escritores estudiados por Casamayor-Cisneros -frente a otros como Leonardo Padura, Pedro Juan Gutiérrez o Antonio José Ponte-, seguimos encontrando en ellos algunos de los temas más frecuentemente utilizados por "los mayores" para representar el derrumbe de la nación o la denuncia social, entre los que destacan, por su amplia utilización, el lugar común de las ruinas o el del hacinamiento. Es cierto que en manos de Ena Lucía Portela (Cien botellas), Wendy Guerra, Yohamna Depestre (D-21), Gerardo Fernández $\mathrm{Fe}$ (La falacia) u Orlando Pardo Lazo (Boring Home) dichos elementos aparecen desideologizados o carentes de carga social; pero, aun así, la ingravidez puede ser vista como una respuesta indirecta (y no una no respuesta) a la decadencia:

La indiferencia ante la sociedad es expresión de una utopía. En los personajes aquí presentados, el permanecer inconexos y flotantes es una idea de la relación colectiva, basada esta vez en el ideal de la no relación. La suspensión ética es la respuesta del sujeto ingrávido ante la situación actual de la sociedad cubana, donde la parálisis política y económica hace de la isla una especie de inquietante marasmo. Nada parece moverse hacia ningún sitio. Muchos cubanos no piensan ya en el Progreso, ni en escapar del subdesarrollo, como se soñaba mientras la revolución representaba un proyecto viable de la modernidad. Ya ni siquiera consiguen ver la decadencia, que se ha vuelto estable a través de más de un decenio de Período Especial. ¿Adónde van los cubanos de la isla hoy en día? (Casamayor-Cisneros 292)

De otro calibre parece ser la indiferencia ante la cubanidad de los autores que comienzan a publicar ya bien entrado el siglo XXI. No se trata tanto de un intento por aniquilar o desentenderse de lo autóctono como de un afán por re- 
definirlo, en parte recurriendo a toda una serie sustancias ajenas, de las que se apropian y con cuya resemantización parecen estar erigiendo una barrera que los separe tanto de la fruta tropical, el café o el azúcar de los abuelos como de las ruinas de los padres. En palabras de Rojas:

Esta es una literatura que se autolocaliza en el después del después, es decir, en el después de la caída del Muro de Berlín, de la desintegración de la URSS, del derribo de las Torres Gemelas y otros hitos finiseculares que marcaron a las generaciones previas. Pero también parece ser una literatura que busca colocar en el detrás de su temporalidad conceptos básicos de la vida cultural y política del último tramo del siglo XX cubano como "revolución", "socialismo" o "transición". Es otro país el que narra esta literatura porque es otro el país que la produce. La decadencia y la ruina acabaron su obra y es preciso narrar las nuevas comunidades con la métrica de una ficción global.

Por su parte, en una entrevista de 2012, Jorge Enrique Lage, que estudió bioquímica, apunta lo siguiente:

De una de las asignaturas del quinto año de la carrera, Toxicología, recuerdo el siguiente precepto: No hay sustancias inocuas, sólo maneras inocuas de utilizar las sustancias. Algo así. Donde dice utilizar podemos poner también mezclar, combinar, agitar [...]. No escribas de manera inocua, me digo hoy a mí mismo, intenta escribir de la manera menos inocua posible. Fracaso la mayoría de las veces, pero es una idea que me persigue: la toxicidad de la ficción. (Grillo)

Más allá de que podamos interpretar sus palabras en la línea que sugiere el entrevistador, a la luz de la alquimia genérica, de los niveles de realidad y de los escenarios, debemos reparar en la importancia que Lage les concede a las sustancias y a los efectos que estas puedan producir en el lector. Lejos de constituir un nuevo coqueteo con el realismo, este apego a la materia se torna una interesante desviación que queda condensada en la imagen de la tabla periódica que funge como espejo en Carbono 14 (una novela de culto).

Publicada en 2010, Carbono 14 marca voluntariamente distancia con la estética realista desde su mismo comienzo: "A falta de otro nombre se llama Evelyn, tiene once o doce años y cayó en La Habana, la misma Habana del realismo, un día cualquiera de cualquier año del siglo XXI. Cuando ya nadie estaba para eso" (5). La protagonista, recién aterrizada en esa Habana distópica de la ficción proveniente del planeta Cuba, enarbola una tabla periódica de elementos químicos que, tras vestirse y acicalarse, le devuelve su reflejo a modo de espejo. La importancia 
de la materia - de las sustancias de que estamos compuestos - no solo queda manifiesta en dicha imagen, sino que se prolonga en la identificación de la tabla periódica con un mapa (145) o con un oráculo desfasado o defectuoso $(41,145)$ o, incluso, en la broma de "empapelar las paredes con segmentos y ampliaciones de tablas periódicas rosadas y floridas" (22). La estrategia de Lage de extremar situaciones e ideas y llevarlas hasta sus últimas consecuencias, revistiéndolas con un barniz de incongruencia e ironía es, junto a la recurrencia a la estética del cómic, la fanfiction y una suerte de ciencia ficción de andar por casa, la clave de su alejamiento del modo de proceder realista. Por eso el interés por los objetos y la recreación constante en ellos y en sus enumeraciones dista de todo realismo. $\mathrm{Al}$ contrario, de forma autoconsciente y metairónica, la novela propone ahondar en la esencia de los objetos de una manera totalmente distinta y grotesca, cuando uno de los protagonistas, JE, adquiere el "KIT RADIOMÉTrICO C-14", "PARA UNA EXACTA MEDICIÓN DE LA ANTIGÜEDAD DE LOS MATERIALES":

Empecé a medir. Empecé, como se dice, a datar. Puertas, muebles, trastos, gomas, cortezas. Se convirtió (me convertí) en una instantánea obsesión. El kit tenía por un lado un sensor que, puesto en contacto con la materia muerta, arrojaba la cifra en menos de un segundo por el otro lado.

Viejos jeans míos, cadavéricos y rasgados en las rodillas, databan del principio de la era del grunge. Un jarrón en forma de huevo que Amanda Peet (a lot like love) le había regalado a mi amigo Frank, resultó ser una reliquia del siglo I (y da igual si antes o después de otra era). Me comí cajas de cereales Kellogg's que no tenían ni dos meses. No sé qué derrame cerebral latía bajo esas mediciones. Pero fuese lo que fuese lo que yo estaba buscando sin saberlo, lo encontré. (54)

A partir de ahí, Lage desarticula el espacio habanero y lo dota de irrealidad mediante un relato carente por completo de lógica y en el que lo grotesco campa a sus anchas. Lejos de practicar en los consabidos yacimientos arqueológicos (las ruinas habaneras), el objeto de las prácticas de datación del protagonista será la ropa interior femenina, que se revela como uno de los elementos más antiguos de la humanidad, así como un sinnúmero de objetos cotidianos.

Rachel Price ha señalado cómo la obra de Lage entronca con autores como Mario Bellatin o César Aira, en su preocupación con la mutabilidad del cuerpo humano y las especies y con el destino de la producción cultural en la era del sampleo. Pero - apunta Price- aunque tanto Carbono 14 como Vultureffect se encuadran en el tipo de ficción contemporánea posnacional global a la que Eloy Fernández Porta ha llamado afterpop, Lage mantiene también un incuestionable armazón cubano, pudiendo su obra ser leída como una meditación sobre la temporalidad en la 
Cuba del siglo XXI al mismo tiempo que como una especulación sobre la Cuba posrevolucionaria o como una pos-Cuba: una ficción, en definitiva, sobre cómo vivir en la isla con una conciencia planetaria. En "Skyline", de Vultureffect (2011), publicado poco después de Carbono 14, se aventura esa posibilidad:

SKYLINE

Escribir La Habana sin el color del verano. Una ciudad en la que estemos ausentes. Poner en ella algo de jerga personal, algo demasiado insoportable y pop, como si toda clase de ficciones extrañas estuvieran a punto de romper. (92)

De ahí la pregunta de Price acerca de qué se data exactamente en la novela (con la conjunción del kit radiométrico del carbono 14 y esa tabla periódica de los elementos, que sirve tanto para decodificar el extinto planeta Cuba como para construir uno nuevo): ¿se trata de una arqueología de un presente cubano lastrado por su pasado o de una arqueología de la era digital global? La lista de las especies humanas extinguidas de las deleted scenes, recogidas en la sección Copy \& Paste del final del libro, apuntaría más bien a la segunda opción: "homo florensis, homo katie holmes, homo neanderthalensis, homo antecesor, homo wii, homo xbox...". (187)

No parece desprovisto de sentido el hecho de que, en la amplia nómina de objetos, materiales y sustancias no tradicionalmente cubanas que con frecuencia aparece en la obra de estos autores, el chicle tenga un lugar de honor. Para autores desinteresados por las esencias cubanas, el artificio de la fruta y el azúcar reunidos en una goma de mascar, resulta - a todas luces - atrayente, máxime cuando se trata de una sustancia enraizada en la cultura popular y el modo de vida occidental. Si en la obra de Lage encontramos vaginas con sabor a chicle (Carbono 14 62) o personajes que lo mascan con irreverencia mientras preguntan si había sicópatas presos en Cuba (155), la goma pasa a convertirse en protagonista absoluta en el poemario Chicle de la camagüeyana Legna Rodríguez Iglesias.

En Chicle (ahora es cuando) es justamente esa sustancia la reina de un poemario en el que se abordan temáticas no necesariamente enraizadas en el presente (el acto mismo de escribir, la sonoridad de la lengua, el tiempo, el suicidio, etc.). La propia escritura muestra, autoconsciente, esa voluntad por sustituir otras sustancias más ancladas en la realidad cubana como estrategia poética:

Creí que me iría bien arañando estas paredes

que me iría de maravillas

mi creencia consistía en sustituir

el lápiz por el bolígrafo

la página en blanco por el poema 


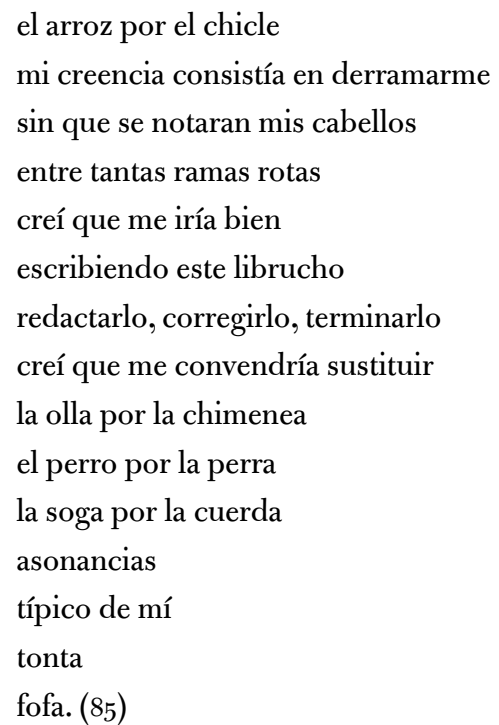

Más allá de la propia sustancia, del chicle se incorpora al poemario incluso su envoltorio, desde el momento en que su sonido se convierte en materia poética: El aborto del chicle no ocurrió por delante ni por detrás ni por la boca fue por una cavidad auditiva que yo nunca entendí como cavidad auditiva porque nunca escuché nada por allí una vez me detuve a escuchar al observar el movimiento de los labios de los otros y no escuché nada por allí quise pero no escuché fue por allí por donde expulsé el chicle al que también pude haber llamado uña fue por allí exactamente por el pliegue que divide al brazo del antebrazo una vez extendí los brazos para escuchar pero no escuché no entendí el movimiento de los labios de los otros hasta que los otros articularon esta palabra: chimenea. (83) 
Con grandes dosis de ironía, Legna Rodríguez acomete la reescritura de una serie de tópicos poéticos despoetizándolos y utilizando el chicle como sustituto de la sustancia poética, dada su condición de "elemento en contacto con la lengua". Esa estructura preciosa que es el chicle (29) le servirá, además, para la reflexión metapoética e, incluso, para ubicarse respecto a la tradición literaria:

Casi al despertar

doblada en los asientos de una Terminal de ómnibus

cuando los clientes que poseen reservación

para el turno de las seis y treinta

abordan su constante medio de transporte

el flujo de poesía es inminente

en mi bolsillo

un viejo chicle de ayer

desde mis uñas

otro poema que nace

saborizado

menta

fresa

melocotón

incorporando mi cuerpo al andén

reprimo el flujo. (35)

De hecho, se produce en torno al chicle lo que podría denominarse un enfrentamiento generacional (aunque las posibilidades de interpretación son mucho más amplias), a partir de la oposición ustedes/nosotros:

Ustedes cierran la verja

cuando nosotros llegamos

inesperadamente

porque ustedes creen que no existe

aquello que nosotros creemos que sí existe.

Ustedes matan los cachorros

que nosotros parimos

por la boca

porque ustedes creen que no puede ser

aquello que nosotros creemos que sí puede ser

aunque no pueda ser.

Ustedes queman los libros

que nosotros leemos 


\author{
sin parar \\ porque ustedes creen \\ que una cosa sustituye a la otra. \\ Ustedes se van quedando \\ boquiabiertos \\ mientras nosotros comenzamos \\ a masticar el chicle. (67)
}

El chicle fungirá, así, como la mayonesa en Mayonesa bien brillante. Una novela de amor (2012) o la piedra en Chupar la piedra (2013), como una sustancia activa en relación con la cual acontecen varias de las funciones del texto y en torno a la que se produce el ensamblaje de la obra. Al final de esta, en el sitio en que habitualmente va el colofón con el lugar de la edición y el número de la tirada (pero en realidad como irónica prolongación de la ficción poética), el lector encuentra los ingredientes del libro: "Goma basé, jarabe, saborizante natural y artificial, ácido cítrico, lícitina, gelatina, goma arábiga, azúcares inconvenientes, almidón modificado". Con una recomendación: "MEJOR COMA FRUTAS Y VERDURAS".

\title{
Conclusiones
}

Considerando lo que Jane Bennett ha llamado thing power, podemos aceptar uno de los planteamientos comunes a la colección de artículos en la que inserta la presente contribución: que en ciertos autores ciertas sustancias adquieren la condición de actantes y cobran una relevancia en los textos en que aparecen que no les viene dada únicamente por el modo en que se relacionan con su entorno textual. Su relevancia en los autores aquí considerados se determina por las relaciones in absentia, es decir, por cómo se apropian de un espacio ajeno, ocupado en la tradición cubana por otros materiales (el arroz, el café, el azúcar, la fruta), para dotarlo de nuevas y renovadas connotaciones. Sin atreverme a hablar de gesto generacional, pues la muestra analizada es demasiado pequeña, sí me parece fructífero analizar la obra de estos y otros autores muy jóvenes en los términos aquí expuestos y ver en esa y otras presencias una estrategia de distanciamiento respecto a las generaciones anteriores.

\section{Obras citadas}

Bennett, Jane. Vibrant Matter: A Political Ecology of Things.

Durham: Duke University Press, 2010. Impreso.

Bauman, Zygmunt. Vida líquida. Barcelona: Paidós, 2006. Impreso. 
Birkenmaier, Anke y Esther Whitfield, eds. Havana Beyond the Ruins: Cultural Mappings after 1989. Durham: Duke University Press, 2011. Impreso.

Casamayor-Cisneros, Odette. Utopía, distopía e ingravidez: reconfiguraciones cosmológicas en la narrativa postsoviética cubana. Madrid: Iberoamericana, 2013. Impreso.

Fernández Porta, Eloy. Afterpop: la literatura de la implosión mediática. Barcelona: Anagrama, 2010. Impreso.

Grillo, Rafael. "La toxicidad de la ficción, una idea que me persigue...". Isliada (9 de julio de 2012). Web. 29 de septiembre de 2015.

Jameson, Fredric. El posmodernismo o la lógica cultural del capitalismo avanzado. Barcelona: Paidós, 1991. Impreso.

Lage, Jorge Enrique. Carbono 14 (una novela de culto). La Habana: Letras Cubanas, 2012. Impreso.

Lage, Jorge Enrique. Vultureffect: cuento. La Habana: Unión, 2011. Impreso.

Loss, Jacqueline y José Manuel Prieto, eds. Caviar with Rum: Cuba-USSR and the Post-Sovietic Experience. New York: Palgrave MacMillan, 2012. Impreso.

Price, Rachel. "Planet/Cuba: On Jorge Enrique Lage's Carbono 14: una novela de culto and Vultureffect". La Habana Elegante (2012). Web. 29 de septiembre de 2015.

Rodríguez Iglesias, Legna. Chicle (ahora es cuando). México D. F.: Literal, 2013. Impreso.

Rodríguez Iglesias, Legna. Chupar la piedra. La Habana: Casa Editora Abril, 2013. Impreso.

Rodríguez Iglesias, Legna. Mayonesa bien brillante: una novela de amor. Matanzas: Ediciones Matanzas, 2012. Impreso.

Rojas, Rafael. "Hacia la ficción global". Libros del Crepúsculo (13 de abril de 2014). Web. 29 de septiembre de 2015.

Rubio, Raúl. "Cosas cubanas: economía, ideología y estética de la cultura material cubana". Espéculo. Revista de Estudios Literarios 44 (2010). Web. 29 de septiembre de 2015.

Rubio, Raúl. "Materializing Havana and Revolution: Cuban Material Culture". Studies in Latin American Popular Culture 24 (2005): 161-77. Impreso. 